\title{
Canagliflozin and Cardiovascular and Renal Events in Type 2 Diabetes
}

\author{
Bruce Neal, M.B., Ch.B., Ph.D., Vlado Perkovic, M.B., B.S., Ph.D., \\ Kenneth W. Mahaffey, M.D., Dick de Zeeuw, M.D., Ph.D., Greg Fulcher, M.D., \\ Ngozi Erondu, M.D., Ph.D., Wayne Shaw, D.S.L., Gordon Law, Ph.D., \\ Mehul Desai, M.D., and David R. Matthews, D.Phil., B.M., B.Ch., \\ for the CANVAS Program Collaborative Group*
}

From the George Institute for Global Health, Faculty of Medicine, UNSW Sydney (B.N., V.P.), the Charles Perkins Centre (B.N.), and the Royal North Shore Hospital (V.P., G.F.), University of Sydney, and the Faculty of Medicine, University of New South Wales (B.N.) - all in Sydney; Imperial College London, London (B.N.), and the Oxford Centre for Diabetes, Endocrinology, and Metabolism and Harris Manchester College, University of Oxford, Oxford (D.R.M.) - both in the United Kingdom; the Stanford Center for Clinical Research, Department of Medicine, Stanford University School of Medicine, Stanford, CA (K.W.M.); the University Medical Center Groningen, University of Groningen, Groningen, the Netherlands (D.Z.); and Janssen Research and Development, Raritan, NJ (N.E., W.S. G.L., M.D.). Address reprint requests to Dr. Neal at the George Institute for Global Health, UNSW Sydney, Level 5, 1 King St., Newtown, NSW 2042, Australia, or at bneal@georgeinstitute.org.au.

*A complete list of investigators in the Canagliflozin Cardiovascular Assessment Study (CANVAS) Program is provided in the Supplementary Appendix, available at NEJM.org.

This article was published on June 12, 2017, at NEJM.org.

N EnglJ Med 2017;377:644-57. DOI: 10.1056/NEJMoal611925

Copyright (c) 2017 Massachusetts Medical Society.

\section{A B STRACT}

\section{BACKGROUND}

Canagliflozin is a sodium-glucose cotransporter 2 inhibitor that reduces glycemia as well as blood pressure, body weight, and albuminuria in people with diabetes. We report the effects of treatment with canagliflozin on cardiovascular, renal, and safety outcomes.

\section{METHODS}

The CANVAS Program integrated data from two trials involving a total of 10,142 participants with type 2 diabetes and high cardiovascular risk. Participants in each trial were randomly assigned to receive canagliflozin or placebo and were followed for a mean of 188.2 weeks. The primary outcome was a composite of death from cardiovascular causes, nonfatal myocardial infarction, or nonfatal stroke.

RESULTS

The mean age of the participants was 63.3 years, $35.8 \%$ were women, the mean duration of diabetes was 13.5 years, and $65.6 \%$ had a history of cardiovascular disease. The rate of the primary outcome was lower with canagliflozin than with placebo (occurring in 26.9 vs. 31.5 participants per 1000 patient-years; hazard ratio, 0.86 ; $95 \%$ confidence interval $[\mathrm{CI}], 0.75$ to 0.97 ; $\mathrm{P}<0.001$ for noninferiority; $\mathrm{P}=0.02$ for superiority). Although on the basis of the prespecified hypothesis testing sequence the renal outcomes are not viewed as statistically significant, the results showed a possible benefit of canagliflozin with respect to the progression of albuminuria (hazard ratio, $0.73 ; 95 \% \mathrm{CI}, 0.67$ to 0.79 ) and the composite outcome of a sustained $40 \%$ reduction in the estimated glomerular filtration rate, the need for renal-replacement therapy, or death from renal causes (hazard ratio, 0.60; $95 \%$ CI, 0.47 to 0.77 ). Adverse reactions were consistent with the previously reported risks associated with canagliflozin except for an increased risk of amputation (6.3 vs. 3.4 participants per 1000 patient-years; hazard ratio, 1.97; 95\% CI, 1.41 to 2.75); amputations were primarily at the level of the toe or metatarsal.

\section{CONCLUSIONS}

In two trials involving patients with type 2 diabetes and an elevated risk of cardiovascular disease, patients treated with canagliflozin had a lower risk of cardiovascular events than those who received placebo but a greater risk of amputation, primarily at the level of the toe or metatarsal. (Funded by Janssen Research and Development; CANVAS and CANVAS-R ClinicalTrials.gov numbers, NCT01032629 and NCT01989754, respectively.) 
T YPE 2 DIABETES MELLITUS IS ASSOCIATed with a substantial risk of cardiovascular and renal disease. ${ }^{1,2}$ The use of inhibitors of sodium-glucose cotransporter 2 (SGLT2) results in favorable effects on biomarkers, including glycemia, blood pressure, weight, ${ }^{3}$ intrarenal hemodynamics, and albuminuria, ${ }^{4}$ and may also reduce the risk of serious cardiovascular complications, kidney disease, and death. ${ }^{5-7}$ The CANVAS Program, comprising two sister trials, was designed to assess the cardiovascular safety and efficacy of canagliflozin and to evaluate the balance between any potential benefits of the drug and the risks associated with it, such as genitourinary infection, diabetic ketoacidosis, and fracture. The Canagliflozin Cardiovascular Assessment Study (CANVAS) ${ }^{8}$ was initiated in December 2009, before the approval of canagliflozin by the Food and Drug Administration (FDA), with the initial goal of showing cardiovascular safety. The first approval of the compound by the FDA occurred in March 2013, with interim data from CANVAS. Owing to the inclusion of unmasked interim cardiovascular outcome data in the regulatory filing documents, a planned expansion of the sample size to enable a test of cardiovascular protection was not undertaken. Instead, CANVAS-Renal (CANVAS-R) ${ }^{9}$ was designed as a second CANVASlike, double-blind, randomized, placebo-controlled trial to be analyzed jointly with CANVAS, to meet a post-approval cardiovascular safety commitment to regulatory agencies. CANVAS-R, which commenced in 2014, was also designed to assess effects on albuminuria. The integrated analysis of CANVAS and CANVAS-R as the CANVAS Program ${ }^{10}$ was undertaken to maximize statistical power to detect plausible effects of canagliflozin on cardiovascular, kidney, and safety outcomes as suggested from evolving evidence about SGLT2 inhibitors. ${ }^{6,7}$

\section{METHODS}

\section{PROGRAM DESIGN AND OVERSIGHT}

A total of 667 centers in 30 countries were involved in the two trials. The trials were scheduled for joint close-out and analysis when at least 688 cardiovascular events had been observed and the last participant who had undergone randomization had approximately 78 weeks of follow-up; this occurred in February 2017. The protocols for the two trials ${ }^{8,9}$ were approved by the ethics committee at each site and are available with the full text of this article at NEJM.org. All the participants provided written informed consent. The trials were sponsored by Janssen Research and Development and were conducted as a collaboration between the sponsor, an academic steering committee, and an academic research organization, George Clinical. Members of the committees are listed in the Supplementary Appendix, available at NEJM.org. Analyses were carried out independently by the sponsor and George Clinical. The first draft of the manuscript was written by the first author, with all coauthors participating in subsequent revisions. MedErgy provided medical writing support, funded by the sponsor. The authors, who had full access to the data and made the final decisions about the content of the manuscript, vouch for the accuracy and completeness of the data and analyses and for the fidelity of the trial to the protocol. The decision to submit the manuscript for publication was made jointly by all the authors.

\section{PARTICIPANTS}

The main criteria for inclusion were identical in the two trials (Table S1 in the Supplementary Appendix). Participants were men and women with type 2 diabetes (glycated hemoglobin level, $\geq 7.0 \%$ and $\leq 10.5 \%$ ) and were either 30 years of age or older with a history of symptomatic atherosclerotic cardiovascular disease or 50 years of age or older with two or more of the following risk factors for cardiovascular disease: duration of diabetes of at least 10 years, systolic blood pressure higher than $140 \mathrm{~mm} \mathrm{Hg}$ while they were receiving one or more antihypertensive agents, current smoking, microalbuminuria or macroalbuminuria, or high-density lipoprotein (HDL) cholesterol level of less than $1 \mathrm{mmol}$ per liter $(38.7 \mathrm{mg}$ per deciliter). Participants were required to have an estimated glomerular filtration rate (eGFR) at entry of more than $30 \mathrm{ml}$ per minute per $1.73 \mathrm{~m}^{2}$ of body-surface area and to meet a range of other criteria.

\section{RANDOMIZATION, TREATMENT, AND FOLLOW-UP}

All potential participants completed a 2-week, single-blind, placebo run-in period. Randomization was performed centrally through an interactive Web-based response system with the use of a computer-generated randomization schedule with randomly permuted blocks that was prepared 
by the trial sponsor. Participants in CANVAS were randomly assigned in a 1:1:1 ratio to receive canagliflozin at a dose of $300 \mathrm{mg}$, canagliflozin at a dose of $100 \mathrm{mg}$, or matching placebo, and participants in CANVAS-R were randomly assigned in a 1:1 ratio to receive canagliflozin, administered at an initial dose of $100 \mathrm{mg}$ daily with an optional increase to $300 \mathrm{mg}$ starting from week 13, or matching placebo. Participants and all trial staff were unaware of the individual treatment assignments until completion of the trial. Use of other background therapy for glycemic management and other control of risk factors were guided by best practice instituted in line with local guidelines.

After randomization, face-to-face follow-up was scheduled in three visits during the first year and at 6-month intervals thereafter, with telephone follow-up between face-to-face assessments. Every follow-up included inquiry about primary and secondary outcome events and serious adverse events. The urinary albumin-to-creatinine ratio was measured every 26 weeks in CANVAS-R and at week 12 and then annually in CANVAS. Measurement of serum creatinine with eGFR was performed at least every 26 weeks in both trials. Participants who prematurely discontinued the trial regimen continued scheduled follow-up whenever possible; extensive efforts were made to obtain full outcome data for all participants during the final follow-up window that spanned November 2016 to February 2017.

\section{OUTCOMES}

The primary outcome was a composite of death from cardiovascular causes, nonfatal myocardial infarction, or nonfatal stroke. Secondary outcomes planned for sequential conditional hypothesis testing were death from any cause, death from cardiovascular causes, progression of albuminuria, and the composite of death from cardiovascular causes and hospitalization for heart failure (Fig. S1 in the Supplementary Appendix). Progression of albuminuria was defined as more than a $30 \%$ increase in albuminuria and a change from either normoalbuminuria to microalbuminuria or macroalbuminuria or from microalbuminuria to macroalbuminuria. If sequential testing was not significant for all the outcomes specified, the remaining outcomes were scheduled for assessment as exploratory variables in the integrated data set.
Exploratory cardiovascular outcomes prespecified for evaluation were nonfatal myocardial infarction, nonfatal stroke, and hospitalization for heart failure, ${ }^{10}$ and the key prespecified exploratory renal outcomes were regression of albuminuria (using criteria comparable to those defined for category progression) and the renal composite comprising a $40 \%$ reduction in eGFR sustained for at least two consecutive measures, the need for renal-replacement therapy (dialysis or transplantation), or death from renal causes (defined as death with a proximate renal cause). Evaluation of total hospitalizations was also prespecified.

All major cardiovascular events, renal outcomes, and deaths, plus selected safety outcomes, were adjudicated by end-point adjudication committees. The members of the committees and the definitions that were used for the clinical events are listed in the Supplementary Appendix. Intermediate markers of cardiovascular risk and requirement for antihyperglycemic agents were assessed to help understand the observed effects on cardiovascular and renal outcomes. Analyses of safety included adverse events coded with the use of the latest version of the Medical Dictionary for Regulatory Activities (MedDRA). For bone fracture, the primary prespecified analysis was for low-trauma fracture events, but secondary analysis of all fractures was also performed. Amputations were assessed overall, but the numbers of cases above and below the ankle were also reported.

\section{STATISTICAL ANALYSIS}

The primary hypothesis test was a test of noninferiority, with the use of a margin of 1.3 for the hazard ratio for the primary outcome with canagliflozin as compared with placebo in the full, integrated data set (i.e., all available follow-up data from all participants who underwent randomization) and with the intention-to-treat approach. We calculated that with 688 cardiovascular safety events recorded across the trials, there would be at least $90 \%$ power, at an alpha level of 0.05 , to exclude an upper margin of the $95 \%$ confidence interval for the hazard ratio of 1.3. Cardiovascular safety was to be shown if the upper boundary of the $95 \%$ confidence interval of the hazard ratio with canagliflozin as compared with placebo was less than 1.3, and superiority was to be shown if the upper boundary was less than 1.0. Hypothesis testing was scheduled to proceed sequentially, conditional on the primary 
safety hypothesis and each subsequent test for superiority being met in the full, integrated data set; in a truncated data set; or in the CANVAS-R data set (Fig. S1 in the Supplementary Appendix). In addition to the formal hypothesis testing, a supplementary set of exploratory analyses of cardiovascular outcomes, renal outcomes, death, and hospitalizations was prespecified for the full, integrated data set with all available follow-up of all participants who underwent randomization. ${ }^{10}$

Hazard ratios, 95\% confidence intervals, and $\mathrm{P}$ values were estimated with Cox regression models ${ }^{11}$ with stratification according to trial and history of cardiovascular disease, for all canagliflozin groups combined versus placebo. P values for efficacy were reported only when the hypothesis was proved. Supplementary analysis with imputation for missing data by multiple imputation was performed for the primary outcome. Hypothesis testing of the other outcomes in the sequence was not performed beyond the first nonsignificant result. For all subsequent outcomes, which were exploratory, reporting was restricted to the hazard ratio estimates and the nominal $95 \%$ confidence intervals. Annualized incidence rates were calculated per 1000 patient-years of follow-up.

The analyses of albuminuria were based on participants with progression or regression on at least one occasion, with a sensitivity analysis performed for those with evidence of sustained progression or regression. Unless otherwise specified, on-treatment analysis (with data from patients who had a safety outcome while they were receiving canagliflozin or placebo or within 30 days after discontinuation of the drug or placebo) was the primary approach used for the safety assessments. The exception was for fracture, amputation, cancer, and diabetic ketoacidosis outcomes, for which analyses included participants who received at least one dose of drug or placebo and had an event at any time during follow-up. Effects of canagliflozin on continuous outcomes were assessed with the use of mixed models ${ }^{12}$ that included all observed longitudinal data and assumed that missing data were missing at random. For all outcome analyses, we tested the homogeneity of treatment effects across the two contributing trials. Analyses were performed with SAS software, version 9.2, and SAS Enterprise Guide, version 7.11.

\section{RESULTS}

\section{PARTICIPANTS}

The two trials involved a total of 10,142 participants, 4330 in CANVAS and 5812 in CANVAS-R. A total of 9734 participants $(96.0 \%)$ completed the trial (i.e., were alive and were assessed for safety and efficacy outcomes during the final follow-up window or died before the final followup). Vital status was confirmed for 10,100 of the 10,142 participants (99.6\%). The mean follow-up was 188.2 weeks, and the median follow-up 126.1 weeks; the length of follow-up was similar in the canagliflozin and placebo groups but was longer in CANVAS (295.9 weeks) than in CANVAS-R (108.0 weeks). A total of $29.2 \%$ of the participants assigned to canagliflozin and $29.9 \%$ of those assigned to placebo discontinued the assigned regimen prematurely. (Additional information on the participants can be found in Figs. S2 and S3 and Tables S2 and S3 in the Supplementary Appendix.)

The mean age of the participants was 63.3 years, $35.8 \%$ were women, the mean duration of diabetes was 13.5 years, the mean eGFR was $76.5 \mathrm{ml}$ per minute per $1.73 \mathrm{~m}^{2}$, and the median urinary albumin-to-creatinine ratio was 12.3 (as measured in milligrams of albumin and grams of creatinine) (Table 1). Among the participants, $22.6 \%$ had microalbuminuria, $7.6 \%$ had macroalbuminuria, and $65.6 \%$ had a history of cardiovascular disease at baseline. A total of $71.4 \%$ of CANVAS-R participants in the canagliflozin group had the dose of canagliflozin increased to $300 \mathrm{mg}$ during the trial. Patients received other appropriate therapies for the management of glycemia and cardiovascular risks (Table 1, and Table S4 in the Supplementary Appendix). Baseline characteristics were balanced in the canagliflozin and placebo groups (Table 1) and were similar across CANVAS and CANVAS-R (Table S5 in the Supplementary Appendix).

\section{INTERMEDIATE MARKERS OF CARDIOVASCULAR RISK}

The mean difference in glycated hemoglobin level between the canagliflozin group and the placebo group was $-0.58 \%$ ( $95 \%$ confidence interval [CI], -0.61 to -0.56 ), the mean difference in body weight was $-1.60 \mathrm{~kg}$ (95\% CI, -1.70 to -1.51$)$, the mean difference in systolic blood pressure was $-3.93 \mathrm{~mm} \mathrm{Hg}$ (95\% CI, -4.30 to -3.56 ), and the mean difference in diastolic blood pressure was $-1.39 \mathrm{~mm} \mathrm{Hg}$ (95\% CI, -1.61 to -1.17$)(\mathrm{P}<0.001$ 


\begin{tabular}{|c|c|c|c|}
\hline Characteristic & $\begin{array}{l}\text { Canagliflozin } \\
(\mathrm{N}=5795)\end{array}$ & $\begin{array}{l}\text { Placebo } \\
\text { ( } N=4347)\end{array}$ & $\begin{array}{c}\text { Total } \\
(\mathrm{N}=10,142) \dagger\end{array}$ \\
\hline Age $-y r$ & $63.2 \pm 8.3$ & $63.4 \pm 8.2$ & $63.3 \pm 8.3$ \\
\hline Female sex - no. (\%) & $2036(35.1)$ & $1597(36.7)$ & $3633(35.8)$ \\
\hline \multicolumn{4}{|l|}{ Race - no. (\%) } \\
\hline White & $4508(77.8)$ & $3436(79.0)$ & $7944(78.3)$ \\
\hline Asian & $777(13.4)$ & 507 (11.7) & $1284(12.7)$ \\
\hline Black & $176(3.0)$ & $160(3.7)$ & $336(3.3)$ \\
\hline Other & $334(5.8)$ & $244(5.6)$ & $578(5.7)$ \\
\hline Current smoker — no. (\%) & $1020(17.6)$ & $786(18.1)$ & $1806(17.8)$ \\
\hline History of hypertension — no. (\%) & $5188(89.5)$ & $3937(90.6)$ & $9125(90.0)$ \\
\hline History of heart failure — no. (\%) & $803(13.9)$ & $658(15.1)$ & $1461(14.4)$ \\
\hline Duration of diabetes $-\mathrm{yr}$ & $13.5 \pm 7.7$ & $13.7 \pm 7.8$ & $13.5 \pm 7.8$ \\
\hline \multicolumn{4}{|l|}{ History of microvascular disease - no. (\%) } \\
\hline Retinopathy & $1203(20.8)$ & $926(21.3)$ & $2129(21.0)$ \\
\hline Nephropathy & $994(17.2)$ & $780(17.9)$ & $1774(17.5)$ \\
\hline Neuropathy & $1787(30.8)$ & $1323(30.4)$ & $3110(30.7)$ \\
\hline \multicolumn{4}{|l|}{ History of atherosclerotic vascular disease - no. (\%)』 } \\
\hline Coronary & $3234(55.8)$ & $2487(57.2)$ & $5721(56.4)$ \\
\hline Cerebrovascular & $1113(19.2)$ & $845(19.4)$ & $1958(19.3)$ \\
\hline Peripheral & $1176(20.3)$ & $937(21.6)$ & $2113(20.8)$ \\
\hline Any & $4127(71.2)$ & $3197(73.5)$ & $7324(72.2)$ \\
\hline History of cardiovascular disease — no. (\%) & $3756(64.8)$ & $2900(66.7)$ & $6656(65.6)$ \\
\hline History of amputation — no. (\%) & $136(2.3)$ & $102(2.3)$ & $238(2.3)$ \\
\hline Body-mass index $\|$ & $31.9 \pm 5.9$ & $32.0 \pm 6.0$ & $32.0 \pm 5.9$ \\
\hline \multicolumn{4}{|l|}{ Blood pressure - $\mathrm{mm} \mathrm{Hg}$} \\
\hline Systolic & $136.4 \pm 15.8$ & $136.9 \pm 15.8$ & $136.6 \pm 15.8$ \\
\hline Diastolic & $77.6 \pm 9.6$ & $77.8 \pm 9.7$ & $77.7 \pm 9.7$ \\
\hline Glycated hemoglobin — \% & $8.2 \pm 0.9$ & $8.2 \pm 0.9$ & $8.2 \pm 0.9$ \\
\hline \multicolumn{4}{|l|}{ Cholesterol - $\mathrm{mmol} /$ liter } \\
\hline Total & $4.4 \pm 1.1$ & $4.4 \pm 1.2$ & $4.4 \pm 1.2$ \\
\hline HDL & $1.2 \pm 0.3$ & $1.2 \pm 0.3$ & $1.2 \pm 0.3$ \\
\hline LDL & $2.3 \pm 0.9$ & $2.3 \pm 0.9$ & $2.3 \pm 0.9$ \\
\hline Ratio of LDL to HDL & $2.0 \pm 0.9$ & $2.0 \pm 0.9$ & $2.0 \pm 0.9$ \\
\hline Triglycerides - mmol/liter & $2.0 \pm 1.3$ & $2.0 \pm 1.5$ & $2.0 \pm 1.4$ \\
\hline $\mathrm{eGFR}-\mathrm{ml} / \mathrm{min} / 1.73 \mathrm{~m} * *$ & $76.7 \pm 20.3$ & $76.2 \pm 20.8$ & $76.5 \pm 20.5$ \\
\hline \multicolumn{4}{|l|}{ Albumin measurements } \\
\hline Median albumin-to-creatinine ratio (interquartile range) & $12.4(6.71-40.9)$ & $12.1(6.57-43.9)$ & $12.3(6.65-42.1)$ \\
\hline Normoalbuminuria — no./total no. (\%) & $4012 / 5740(69.9)$ & $2995 / 4293(69.8)$ & $7007 / 10,033(69.8)$ \\
\hline Microalbuminuria — no./total no. (\%) & $1322 / 5740(23.0)$ & $944 / 4293(22.0)$ & $2266 / 10,033(22.6)$ \\
\hline Macroalbuminuria - no./total no. (\%) & $406 / 5740(7.1)$ & $354 / 4293(8.2)$ & $760 / 10,033(7.6)$ \\
\hline
\end{tabular}

* Plus-minus values are means \pm SD. The CANVAS Program comprised two trials: the Canagliflozin Cardiovascular Assessment Study (CANVAS) and CANVAS-Renal (CANVAS-R).

$\dagger$ One participant underwent randomization at two different sites; only the first randomization is included in the intention-to-treat analysis set.

$\mp$ Race was determined by investigator inquiry of the participant. Other includes American Indian or Alaska Native, Native Hawaiian or other Pacific Islander, multiple races, other race, and unknown.

$\int$ Some participants had more than one type of atherosclerotic disease.

I A history of cardiovascular disease was defined as a history of symptomatic atherosclerotic vascular disease (coronary, cerebrovascular, or peripheral).

|| The body-mass index is the weight in kilograms divided by the square of the height in meters.

*w. Values for estimated glomerular filtration rate (eGFR) were calculated with data from 5794 participants in the canagliflozin group, 4346 in the placebo group, and 10,140 in the total population.

$\uparrow \dagger$ The albumin-to-creatinine ratio was measured in milligrams of albumin and grams of creatinine. 
for all comparisons) (Fig. 1). The use of other antihyperglycemic agents during follow-up was $9.3 \%$ lower ( $95 \%$ CI, -11.0 to -7.6 ) in the canagliflozin group than in the placebo group. The level of HDL cholesterol was higher in the canagliflozin group than in the placebo group (by $2.05 \mathrm{mg}$ per deciliter; $95 \% \mathrm{CI}, 1.77$ to 2.33 [0.05 mmol per liter; $95 \%$ CI, 0.05 to 0.06$]$ ), as was the level of low-density lipoprotein (LDL) cholesterol (by $4.68 \mathrm{mg}$ per deciliter; 95\% CI, 3.64 to 5.73 [0.12 mmol per liter; 95\% CI, 0.09 to 0.15$]$ ]); the ratio of LDL cholesterol to HDL cholesterol was unchanged (Fig. S4 in the Supplementary Appendix).

\section{CARDIOVASCULAR OUTCOMES, DEATH, AND HOSPITALIZATIONS}

Significantly fewer participants in the canagliflozin group than in the placebo group had a primary outcome event (the composite of death from cardiovascular causes, nonfatal myocardial infarction, or nonfatal stroke): 26.9 vs. 31.5 participants with an event per 1000 patient-years (hazard ratio, 0.86; $95 \%$ CI, 0.75 to 0.97 ; $\mathrm{P}<0.001$ for noninferiority; $\mathrm{P}=0.02$ for superiority) (Figs. 2 and 3). The effects on the primary outcome were nearly the same when imputation for missing events was performed (hazard ratio, 0.85 ; 95\% CI, 0.75 to 0.97). There were broadly consistent effects across a wide range of prespecified subgroups (Fig. 4), except for subgroups defined according to baseline use of diuretics ( $\mathrm{P}<0.001$ for homogeneity). Superiority was not shown for the first secondary outcome in the testing sequence (death from any cause; $\mathrm{P}=0.24$ ), and hypothesis testing was discontinued. Therefore, estimates for the fatal secondary outcomes, including death from any cause (hazard ratio, $0.87 ; 95 \% \mathrm{CI}, 0.74$ to 1.01 ) and death from cardiovascular causes (hazard ratio, $0.87 ; 95 \% \mathrm{CI}, 0.72$ to 1.06 ), are not considered to be significant (Figs. 2, 3, and 5). Effect estimates for exploratory cardiovascular outcomes are shown in Figures 2, 3, and 5. There was no evidence of differences in effects between the CANVAS and CANVAS-R trials for the primary, fatal, or exploratory cardiovascular outcomes (Table S6 in the Supplementary Appendix).

\section{RENAL OUTCOMES}

Progression of albuminuria occurred less frequently among participants assigned to canagliflozin than among those assigned to placebo (89.4 vs. 128.7 participants with an event per 1000 patient- years), corresponding to a hazard ratio of 0.73 (95\% CI, 0.67 to 0.79) (Figs. 3 and 5); the effects were greater in CANVAS-R (hazard ratio, 0.64; 95\% CI, 0.57 to 0.73 ) than in CANVAS (hazard ratio, $0.80 ; 95 \% \mathrm{CI}, 0.72$ to 0.90$)(\mathrm{P}=0.02$ for homogeneity) (Table S7 in the Supplementary Appendix). Regression of albuminuria also occurred more frequently among those assigned to canagliflozin than among those assigned to placebo (293.4 vs. 187.5 participants with regression per 1000 patient-years; hazard ratio, 1.70; 95\% CI, 1.51 to 1.91). The composite outcome of sustained $40 \%$ reduction in eGFR, the need for renal-replacement therapy, or death from renal causes occurred less frequently among participants in the canagliflozin group than among those in the placebo group (5.5 vs. 9.0 participants with the outcome per 1000 patient-years, corresponding to a hazard ratio of $0.60 ; 95 \%$ CI, 0.47 to 0.77 ) (Figs. 3 and 5); no significant difference in this outcome was seen between CANVAS and CANVAS-R (Table S7 in the Supplementary Appendix).

\section{SAFETY OUTCOMES}

Serious adverse events were less common among participants assigned to canagliflozin than among those assigned to placebo (104.3 vs. 120.0 participants with an event per 1000 patient-years; hazard ratio, $0.93 ; 95 \% \mathrm{CI}, 0.87$ to 1.00 ). Adverse events leading to discontinuation did not differ significantly between groups (35.5 vs. 32.8 participants with an event per 1000 patient-years; hazard ratio, 1.13; 95\% CI, 0.99 to 1.28 ) (Table 2). There was a higher risk of amputation of toes, feet, or legs with canagliflozin than with placebo (6.3 vs. 3.4 participants with amputation per 1000 patient-years, corresponding to a hazard ratio of $1.97 ; 95 \%$ CI, 1.41 to 2.75 ), with $71 \%$ of the affected participants having their highest amputation at the level of the toe or metatarsal (Fig. S5 in the Supplementary Appendix). The highest absolute risk of amputation occurred among patients who had a history of amputation or peripheral vascular disease, but the relative risk of amputation with canagliflozin as compared with placebo was similar across these subgroups (Table S8 in the Supplementary Appendix).

Previously reported risks of adverse events of interest attributable to infections of male or female genitalia, volume depletion, and diuresis were observed. We detected no higher risks of hypoglycemia, hyperkalemia, acute kidney injury, pancreatitis, malignancies, or venous thromboembolism 


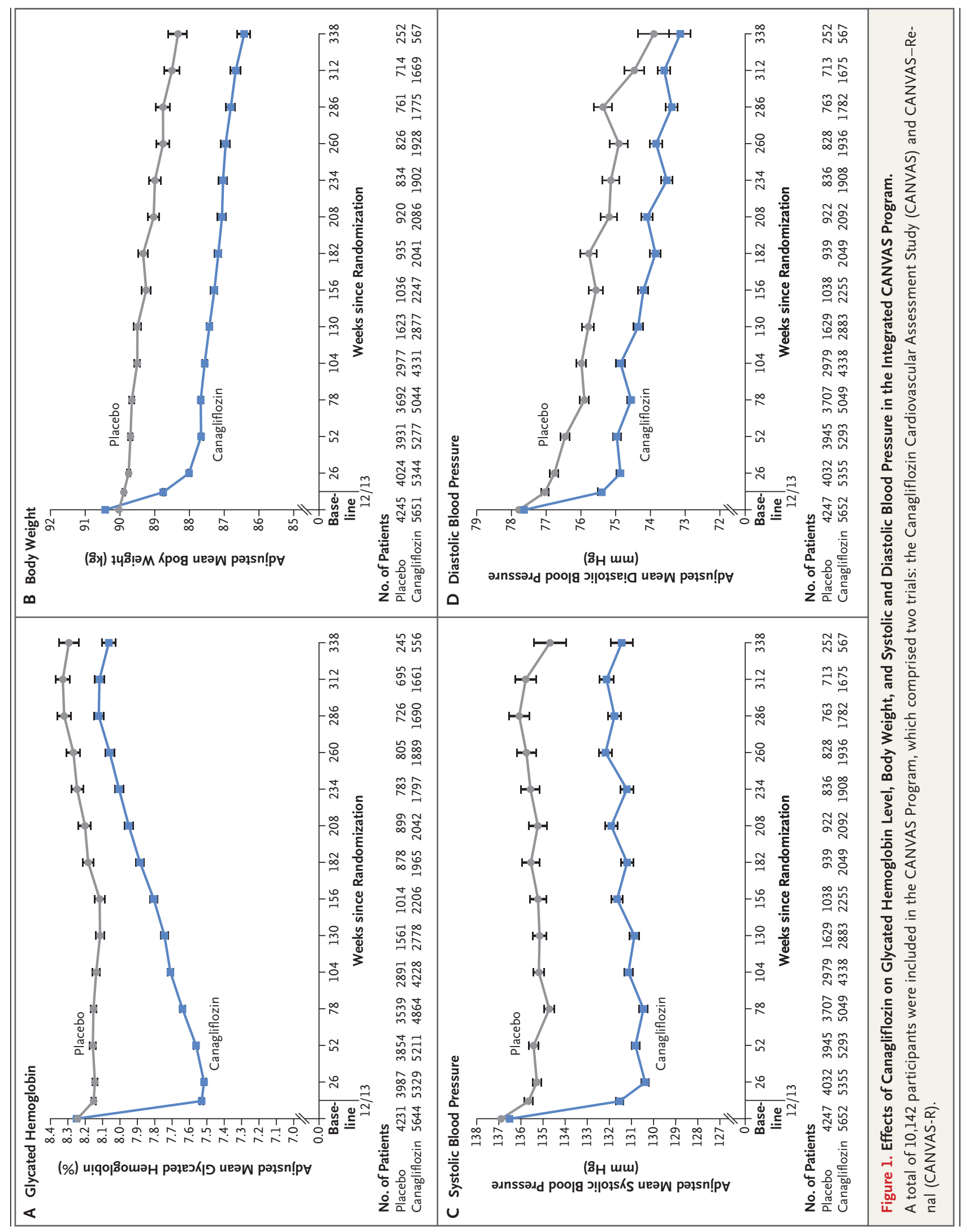




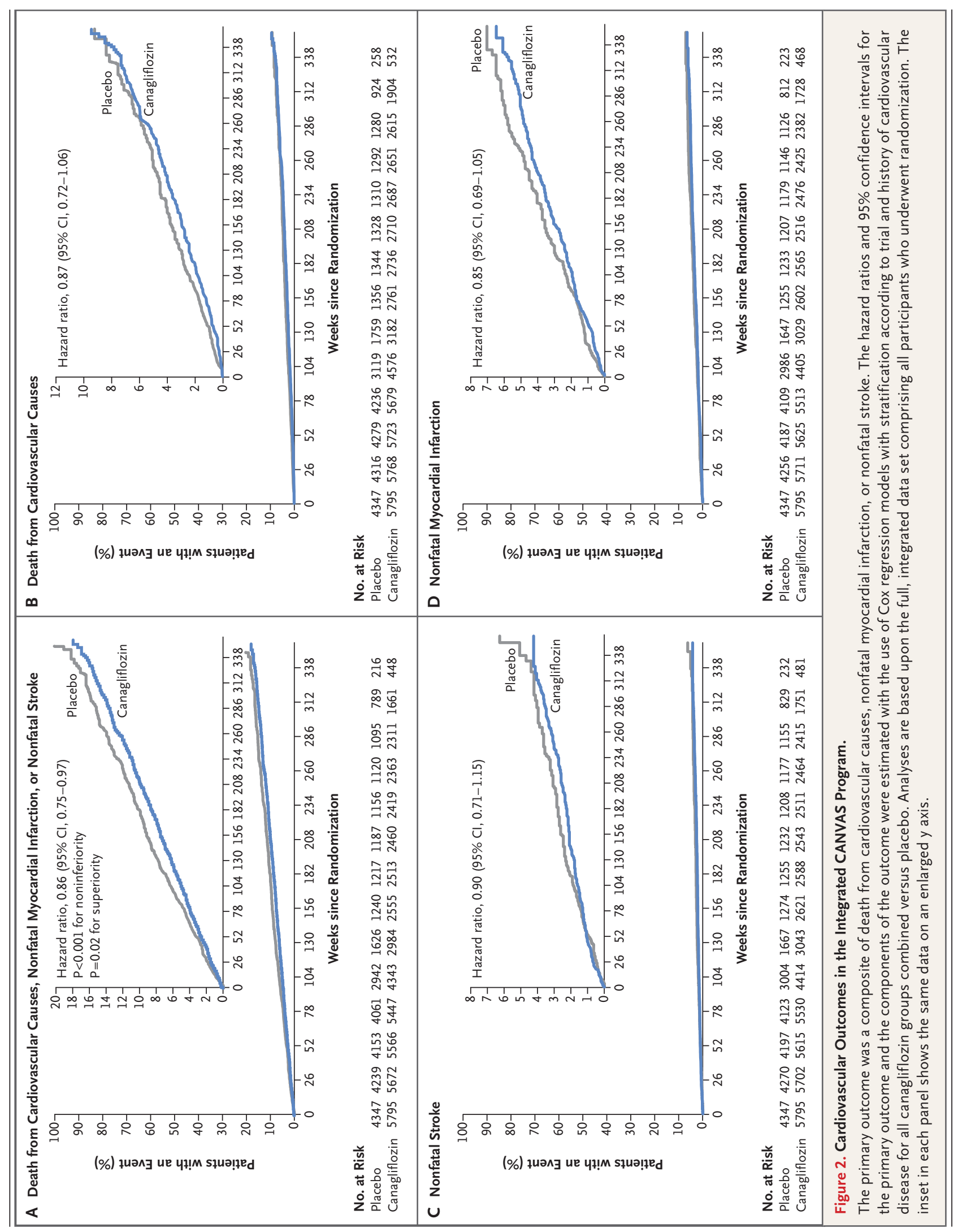

N ENGLJ MED 377;7 NEJM.ORG AUGUST 17, 2017 


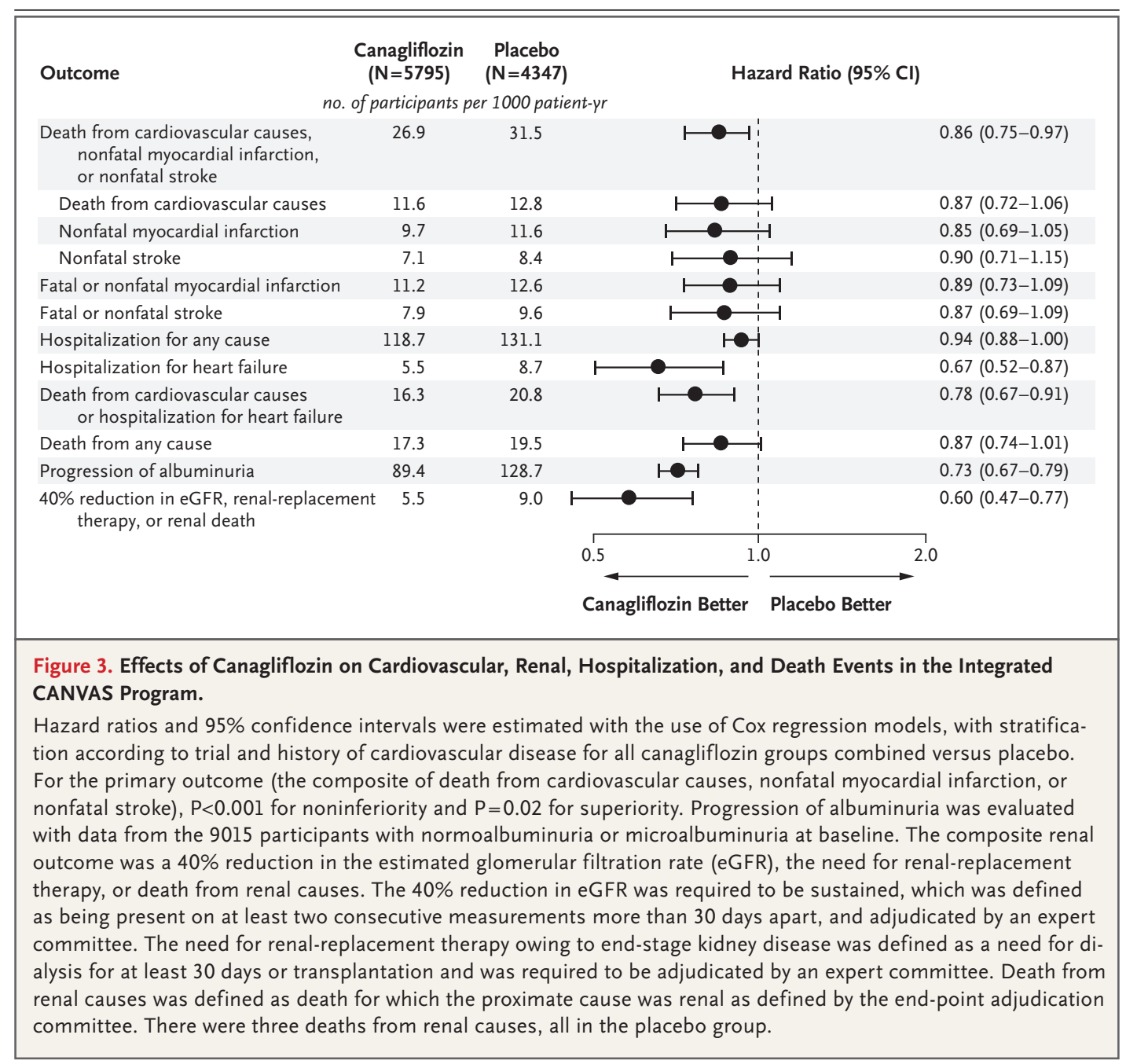

with canagliflozin than with placebo. The rate of all fractures was higher with canagliflozin than with placebo (15.4 vs. 11.9 participants with fracture per 1000 patient-years; hazard ratio, 1.26; $95 \% \mathrm{CI}, 1.04$ to 1.52 ), and there was a similar trend with respect to low-trauma fracture events (11.6 vs. 9.2 participants with fracture per 1000 patient-years; hazard ratio, $1.23 ; 95 \% \mathrm{CI}, 0.99$ to 1.52). There was evidence of heterogeneity in the bone-fracture findings between CANVAS and CANVAS-R with respect to both low-trauma fracture and all fracture (both $\mathrm{P} \leq 0.005$ ), with risks higher in the canagliflozin group than in the placebo group in CANVAS but not in CANVAS-R (Table S9 in the Supplementary Appendix). Only a small number of events of diabetic ketoacidosis were observed with canagliflozin and placebo $(0.6$ vs. 0.3 participants with an event per 1000 patientyears; hazard ratio, 2.33 ; $95 \%$ CI, 0.76 to 7.17 ).

\section{DISCUSSION}

Patients with type 2 diabetes and established cardiovascular disease or at high risk for cardiovas-

Figure 4 (facing page). Effects of Canagliflozin on the Primary Cardiovascular Outcome in Subgroups.

A total of 10,142 participants were included in the analysis. Hazard ratios and $95 \%$ confidence intervals were estimated with the use of Cox regression models. $\mathrm{P}$ values for homogeneity were obtained by fitting interaction terms. The body-mass index (BMI) is the weight in kilograms divided by the square of the height in meters. The analysis according to the history of heart failure was not prespecified. RAAS denotes renin-angiotensin-aldosterone system. 


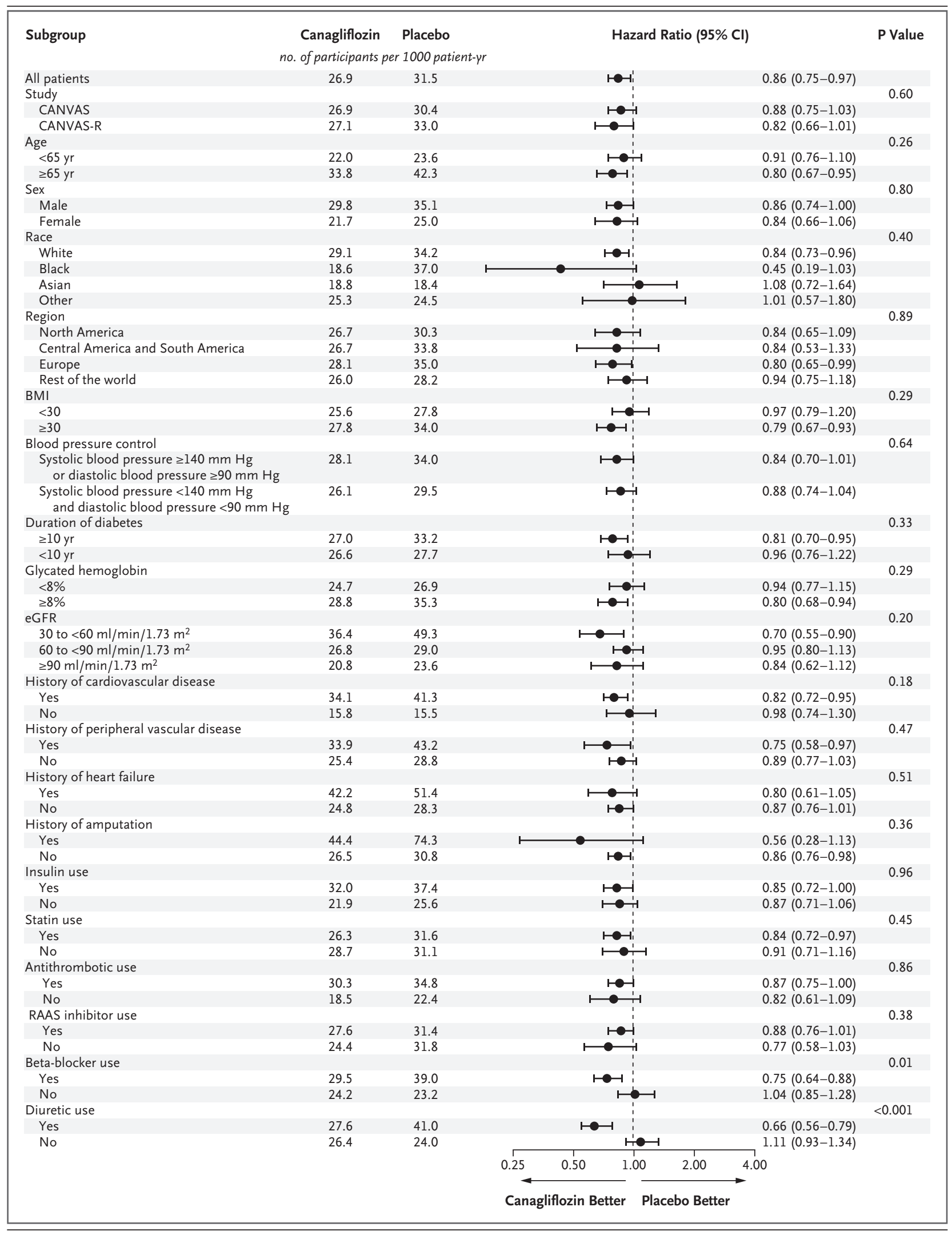

The New England Journal of Medicine 


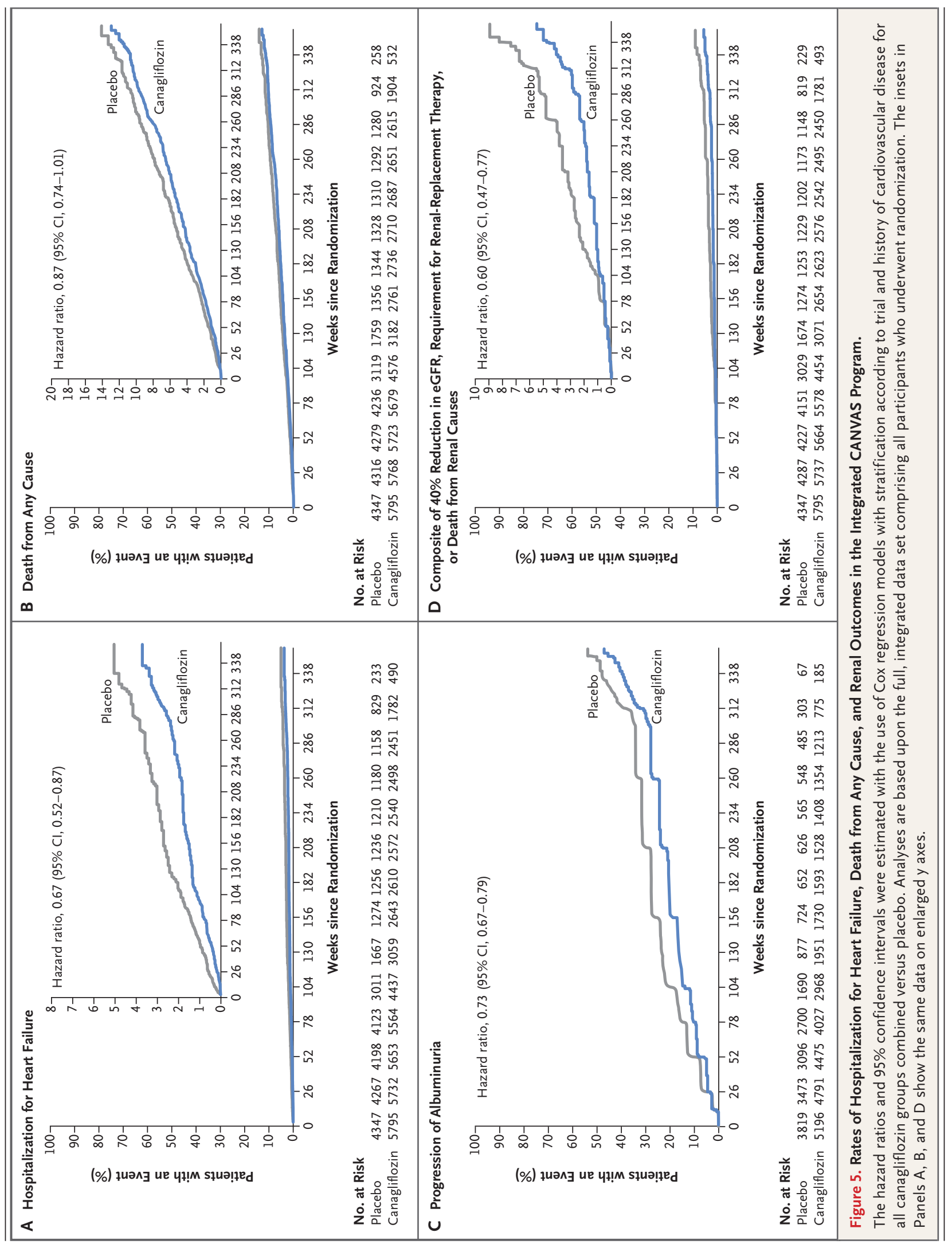




\begin{tabular}{|c|c|c|c|}
\hline Event & $\begin{array}{l}\text { Canagliflozin } \\
\text { event rate } p\end{array}$ & $\begin{array}{l}\text { Placebo } \\
\text { tient-yr }\end{array}$ & P Value ${ }^{\prime}$ \\
\hline All serious adverse events & 104.3 & 120.0 & 0.04 \\
\hline Adverse events leading to discontinuation & 35.5 & 32.8 & 0.07 \\
\hline \multicolumn{4}{|l|}{$\begin{array}{c}\text { Serious and nonserious adverse events of interest } \\
\text { recorded in the CANVAS Program }\end{array}$} \\
\hline Acute pancreatitis (adjudicated) & 0.5 & 0.4 & 0.63 \\
\hline \multicolumn{4}{|l|}{ Cancer } \\
\hline Renal cell & 0.6 & 0.2 & 0.17 \\
\hline Bladder & 1.0 & 1.1 & 0.74 \\
\hline Breast & 3.1 & 2.6 & 0.65 \\
\hline Photosensitivity & 1.0 & 0.3 & 0.07 \\
\hline Diabetic ketoacidosis (adjudicated) & 0.6 & 0.3 & 0.14 \\
\hline Amputation & 6.3 & 3.4 & $<0.001$ \\
\hline \multicolumn{4}{|l|}{ Fracture (adjudicated) $\ddagger$} \\
\hline All & 15.4 & 11.9 & 0.02 \\
\hline Low-trauma & 11.6 & 9.2 & 0.06 \\
\hline Venous thromboembolic events & 1.7 & 1.7 & 0.63 \\
\hline 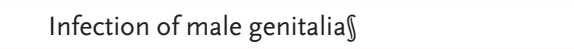 & 34.9 & 10.8 & $<0.001$ \\
\hline \multicolumn{4}{|l|}{$\begin{array}{l}\text { Serious and nonserious adverse events of interest } \\
\text { collected in CANVAS alone }\end{array}$} \\
\hline Osmotic diuresis & 34.5 & 13.3 & $<0.001$ \\
\hline Volume depletion & 26.0 & 18.5 & 0.009 \\
\hline Hypoglycemia & 50.0 & 46.4 & 0.20 \\
\hline Acute kidney injury & 3.0 & 4.1 & 0.33 \\
\hline Hyperkalemia & 6.9 & 4.4 & 0.10 \\
\hline Urinary tract infection & 40.0 & 37.0 & 0.38 \\
\hline Mycotic genital infection in women & 68.8 & 17.5 & $<0.001$ \\
\hline Severe hypersensitivity or cutaneous reaction & 8.5 & 6.1 & 0.17 \\
\hline Hepatic injury & 7.4 & 9.1 & 0.35 \\
\hline Renal-related (including acute kidney injury) & 19.7 & 17.4 & 0.32 \\
\hline
\end{tabular}

* Analyses were performed on data from the on-treatment data set (patients who had a safety outcome while they were receiving canagliflozin or placebo or within 30 days after discontinuation of the drug or placebo), except for fracture, amputation, cancer, and diabetic ketoacidosis outcomes, which included all events at any time point in all patients who underwent randomization and received at least one dose of canagliflozin or placebo.

$\dagger P$ values were estimated from Cox regression models.

Low-trauma fracture was the prespecified primary fracture outcome, and all fracture was a secondary outcome.

$\int$ Infection of male genitalia included balanitis, phimosis, and events leading to circumcision.

If For these adverse events, the annualized incidence rates are reported with data from CANVAS alone through January 7 , 2014, because after this time, only serious adverse events or adverse events leading to discontinuation were collected. In CANVAS-R, only serious adverse events or adverse events leading to discontinuation were collected. Owing to the differences between the two trials in methods of collection of the data, an integrated analysis of these adverse events is not possible.

The New England Journal of Medicine 
cular events who were treated with canagliflozin had significantly lower rates of the primary cardiovascular outcome than patients assigned to placebo. All three components of the primary outcome - death from cardiovascular causes, nonfatal myocardial infarction, and nonfatal stroke - showed point estimates of effect that suggested benefit, although the individual effects did not reach significance. The results also showed that patients treated with canagliflozin had a lower risk of hospitalization for heart failure, progression of albuminuria, and substantive loss of kidney function than patients who received placebo, although on the basis of the prespecified hypothesis testing sequence these findings are not considered statistically significant.

Several established effects of SGLT2 inhibitors on intermediate outcomes may contribute to cardiovascular and renal protection. Although pleiotropic effects have been inferred, improved glycemic control, lowering of blood pressure, decrease in intraglomerular pressure, reduction in albuminuria, and amelioration of volume overload are all plausible protective mechanisms. ${ }^{4,13-23}$

The effects on cardiovascular and renal outcomes observed within this program of two integrated trials are similar to those observed previously with this drug class. ${ }^{5,6}$ The favorable direction of effect with respect to stroke observed in this trial program differs from a previously reported possible adverse effect on stroke risk. ${ }^{5,6}$ Apparent differences in the effect sizes for other secondary and exploratory outcomes may be attributable to chance, since precision in the effect estimates of each is limited. Although it is also possible that variation in effect sizes could reflect features of trial design or actual differences between the agents, additional data will be required to clarify the differing results.

The possible benefit of canagliflozin use with respect to renal outcomes is supported by the magnitude of the effects observed, the consistency of the observation across renal outcomes, and the consistency of the findings with other recently reported data. ${ }^{12}$ Prespecification, confirmation, and adjudication of the renal outcomes in this trial program add support to the possibility that SGLT2 inhibition may have an important kidneyprotective effect in type 2 diabetes. Since most renal events were based on changes in eGFR, more data are required to confirm the effects on kidney failure. Definitive evidence about the effects of canagliflozin on clinical kidney outcomes are likely to be provided by the ongoing Canagliflozin and Renal Endpoints in Diabetes with Established Nephropathy Clinical Evaluation trial (CREDENCE; ClinicalTrials.gov number, NCT02065791).

The adverse effects observed in this program of two integrated trials are generally consistent with the known safety profile of canagliflozin and other SGLT2 inhibitors. ${ }^{5}$ The increased rate of amputation is a new finding for which the mechanism is unknown, and care is warranted in the use of canagliflozin in patients at risk for amputation. An increase in bone fractures has been described previously with canagliflozin ${ }^{24}$ but the excess in bone fracture seen in CANVAS was not observed in CANVAS-R. There is no clear explanation for the difference in fracture risk between the two trials, which included directly comparable patient groups and assessed the same intervention.

The trial program has certain strengths. It benefits from the large size of the combined trials, the long duration of the trials, the randomized design, the breadth of included participants, and the high standard to which the conduct of the trials was held. The inclusion of participants with and those without established cardiovascular disease extends the population for which data are available.

The trial program has certain limitations, the major one being the moderate number of events for many important outcomes - in particular, the paucity of events of end-stage kidney disease. The relatively small proportion of participants with established kidney disease also limits generalization to that population. The large number of analyses and relatively few events increases the risk of false positive findings, though the broad internal and external consistency of the data strengthens the conclusions. Discontinuation of randomized therapy and greater use of other glucose-lowering agents in the placebo group during the trial program is the likely explanation for the convergence of glycated hemoglobin levels over time and may have resulted in underestimation of any benefits and risks associated with canagliflozin.

In summary, the trial program showed that among patients with type 2 diabetes who had an increased risk of cardiovascular disease, patients treated with canagliflozin had a significantly lower risk of death from cardiovascular causes, 
nonfatal myocardial infarction, or nonfatal stroke than those who received placebo but a greater risk of amputation.

Supported by Janssen Research and Development.

Disclosure forms provided by the authors are available with the full text of this article at NEJM.org.

We thank all investigators, trial teams, and patients for participating in these trials and the following people for their contributions to the statistical monitoring and analyses and the protocol development, safety monitoring, and operational implementation over the duration of the two trials: Lyndal Hones, Lucy Perry, Sharon Dunkley, Tao Sun, Hsiaowei Deng, George Capuano, Qiang Li, Severine Bompoint, Laurent Billot, Mary Lee, Joan Lind, Roger Simpson, Mary Kavalam, Terry Barrett, Ed Connell, Michele Wells, Jacqueline Yee, Dainius Balis, Frank Vercruysse, Elisa Fabbrini, Richard Oh, Nicole Meyers, Gary Meininger, and Norm Rosenthal. Medical writing support was provided by Kimberly Dittmar, Ph.D., of MedErgy, and was funded by Janssen Global Services.

\section{REFERENCES}

1. Liyanage T, Ninomiya $\mathrm{T}$, Jha $\mathrm{V}$, et al. Worldwide access to treatment for endstage kidney disease: a systematic review. Lancet 2015;385:1975-82.

2. Whiting DR, Guariguata L, Weil C, Shaw J. IDF diabetes atlas: global estimates of the prevalence of diabetes for 2011 and 2030. Diabetes Res Clin Pract 2011;94:311-21.

3. Vasilakou D, Karagiannis T, Athanasiadou E, et al. Sodium-glucose cotransporter 2 inhibitors for type 2 diabetes: a systematic review and meta-analysis. Ann Intern Med 2013;159:262-74.

4. Cherney DZ, Perkins BA, Soleymanlou N, et al. Renal hemodynamic effect of sodium-glucose cotransporter 2 inhibition in patients with type 1 diabetes mellitus. Circulation 2014;129:587-97.

5. Wu JH, Foote C, Blomster J, et al. Effects of sodium-glucose cotransporter-2 inhibitors on cardiovascular events, death, and major safety outcomes in adults with type 2 diabetes: a systematic review and meta-analysis. Lancet Diabetes Endocrinol 2016;4:411-9.

6. Zinman B, Wanner C, Lachin JM, et al. Empagliflozin, cardiovascular outcomes, and mortality in type 2 diabetes. $\mathrm{N}$ Engl J Med 2015;373:2117-28.

7. Wanner C, Inzucchi SE, Lachin JM, et al. Empagliflozin and progression of kidney disease in type 2 diabetes. $\mathrm{N}$ Engl J Med 2016;375:323-34.

8. Neal B, Perkovic V, de Zeeuw D, et al. Rationale, design, and baseline characteristics of the Canagliflozin Cardiovascular Assessment Study (CANVAS) - a randomized placebo-controlled trial. Am Heart J 2013;166(2):217-223.e11.
9. Neal B, Perkovic V, Matthews DR, et al. Rationale, design and baseline characteristics of the CANagliflozin cardioVascular Assessment Study-Renal (CANVAS-R): a randomized, placebocontrolled trial. Diabetes Obes Metab 2017;19:387-93.

10. Neal B, Perkovic V, Mahaffey KW, et al. Optimizing the analysis strategy for the CANVAS Program: a prespecified plan for the integrated analyses of the CANVAS and CANVAS-R trials. Diabetes Obes Metab 2017 February 28 (Epub ahead of print).

11. Cox DR. Regression models and life tables (with discussion). J R Stat Soc [Ser B] 1972;34:187-220.

12. Verbeke G, Molenberghs G. Linear mixed models for longitudinal data. New York: Springer, 2000.

13. Rabelink TJ, de Zeeuw D. The glycocalyx - linking albuminuria with renal and cardiovascular disease. Nat Rev Nephrol 2015;11:667-76.

14. Ballermann BJ, Brenner BM. Atrial natriuretic peptide and the kidney. Am J Kidney Dis 1987;10:Suppl 1:7-12.

15. Emdin CA, Rahimi K, Neal B, Callender T, Perkovic V, Patel A. Blood pressure lowering in type 2 diabetes: a systematic review and meta-analysis. JAMA 2015; 313:603-15.

16. Lv J, Ehteshami P, Sarnak MJ, et al. Effects of intensive blood pressure lowering on the progression of chronic kidney disease: a systematic review and metaanalysis. CMAJ 2013;185:949-57.

17. Ortola FV, Ballermann BJ, Anderson S, Mendez RE, Brenner BM. Elevated plasma atrial natriuretic peptide levels in dia- betic rats: potential mediator of hyperfiltration. J Clin Invest 1987;80:670-4. 18. Perkovic V, Heerspink HL, Chalmers $\mathrm{J}$, et al. Intensive glucose control improves kidney outcomes in patients with type 2 diabetes. Kidney Int 2013;83:517-23. 19. Stratton IM, Adler AI, Neil HA, et al. Association of glycaemia with macrovascular and microvascular complications of type 2 diabetes (UKPDS 35): prospective observational study. BMJ 2000;321:40512.

20. Vallianou NG, Geladari E, Kazazis CE. SGLT-2 inhibitors: their pleiotropic properties. Diabetes Metab Syndr 2016 December 9 (Epub ahead of print).

21. Wong MG, Perkovic V, Chalmers J, et al. Long-term benefits of intensive glucose control for preventing end-stage kidney disease: ADVANCE-ON. Diabetes Care 2016;39:694-700.

22. Xie X, Atkins E, Lv J, et al. Effects of intensive blood pressure lowering on cardiovascular and renal outcomes: updated systematic review and meta-analysis. Lancet 2016;387:435-43.

23. Zoungas S, Arima H, Gerstein HC, et al. Effects of intensive glucose control on microvascular outcomes in patients with type 2 diabetes: a meta-analysis of individual participant data from randomised controlled trials. Lancet Diabetes Endocrinol 2017;5:431-7.

24. Watts NB, Bilezikian JP, Usiskin $K$, et al. Effects of canagliflozin on fracture risk in patients with type 2 diabetes mellitus. J Clin Endocrinol Metab 2016;101: $157-66$.

Copyright $\odot 2017$ Massachusetts Medical Society. 